\title{
Insulin does not reverse bupivacaine-induced cardiac systole in vitro: the importance of drug elimination to be resuscitated
}

\author{
Wyun Kon Park' ${ }^{1}$, Hyun Joo Kim ${ }^{1}$, Ja Rang Jung ${ }^{1}$, and Lynch C III ${ }^{2}$
}

${ }^{1}$ Department of Anesthesiology \& Pain Medicine, Yonsei University College of Medicine, Seoul, Korea,

${ }^{2}$ Department of Anesthesiology, University of Virginia Health System, Charlottesville, Virginia, USA

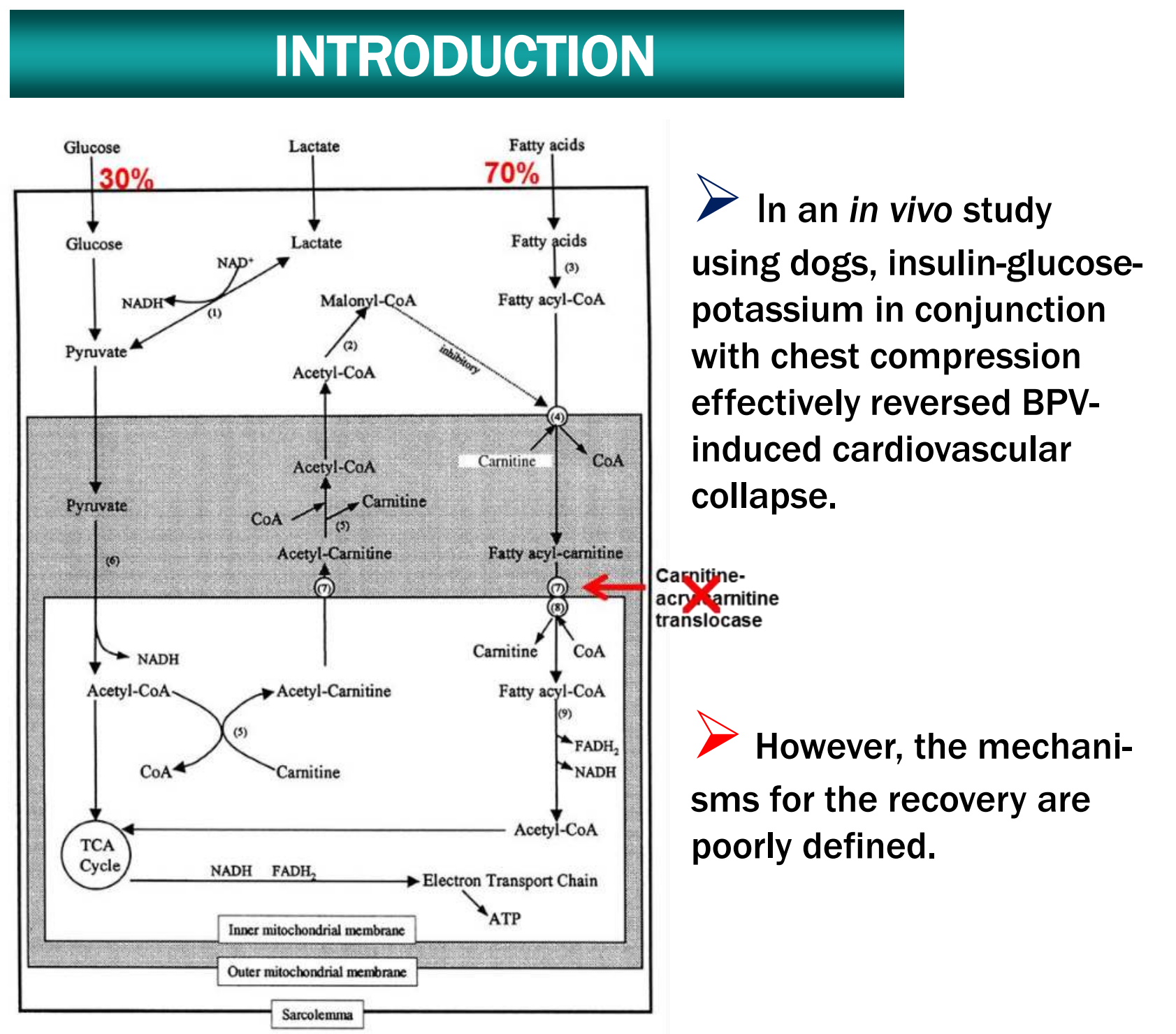

\section{MATERIALS and METHODS}

\section{Preparation of Cardiac Muscles}

- 400-450 g male guinea pigs

- Excision of a Rt. ventricular papillary muscle under SEVO anesthesia

- Mounted horizontally in a tissue bath superfused at $37^{\circ} \mathrm{C}$ with normal Tyrode solution which was recirculated through The bath from a reservoir containing $100 \mathrm{ml}$ of Tyrode solution through with $95 \% \mathrm{O}_{2}$ and $5 \% \mathrm{CO}_{2}$ were bubbled maintaining a $\mathrm{pH}$ of $7.4 \pm 0.05$.

- GRASS F03 force transducer - Field stimulation using a Grass S44 stimulator $\quad$ Powerlab ${ }^{\circledR}$ Data analysis system

\section{Experimental Protocols}

\section{Effects of Insulin}

Cumulative concentration-responses to

$>$ Insulin (Humulin insulin R): $10^{-3}, 10^{-2}, 10^{-1}, 2 \times 10^{-1}, 5 \times 10^{-1}$ units $/ \mathrm{ml}$

II. Recirculation Experiments - continuing BPV

1. Stable contractions at $1.2 \mathrm{~Hz} \rightarrow 50 \mu \mathrm{M} \mathrm{BPV} \rightarrow 10^{-3}, 10^{-2}, 10^{-1}, 2 \times 10^{-1}$, $5 \times 10^{-1}$ units/ml insulin

2. Stable contractions at $1.2 \mathrm{~Hz} \rightarrow$ asystole with $500 \mu \mathrm{M} \mathrm{BPV} \rightarrow 10^{-1}, 2$ $\mathrm{x} 10^{-1}, 5 \times 10^{-1}$ units $/ \mathrm{ml}$ insulin

3. Stable contractions at $1.2 \mathrm{~Hz} \rightarrow$ asystole with $500 \mu \mathrm{M} \mathrm{BPV} \rightarrow 33 \mathrm{mM}$ glucose at $5 \mathrm{~min}$ after asystole for $10 \mathrm{~min} \rightarrow 10^{-1}, 2 \times 10^{-1}, 5 \times 10^{-1}$ units/ml insulin

4. Stable contractions at $1.2 \mathrm{~Hz} \rightarrow 200-300 \mu \mathrm{M} \mathrm{BPV} \rightarrow$ Intermittent contractile responses (conduction block) $\rightarrow 10^{-1}, 2 \times 10^{-1}, 5 \times 10^{-1}$ units/ml insulin

5. Stable contractions at $1.2 \mathrm{~Hz} \rightarrow 200-300 \mu \mathrm{M} \mathrm{BPV} \rightarrow$ conduction block $\rightarrow 33 \mathrm{mM}$ glucose at $5 \mathrm{~min}$ after conduction block for $10 \mathrm{~min} \rightarrow 10^{-1}$, $2 \times 10^{-1}, 5 \times 10^{-1}$ units $/ \mathrm{ml}$ insulin

\section{Measurements of BPV Concentrations in Solution}

Measured under recirculating conditions at $5 \mathrm{~min}$ after asystole, 15, 30, 45 , and $60 \mathrm{~min}$ in either control solution or the control solution
III. Non-recirculation Experiments - BPV washout

1. stable contractions at $1.2 \mathrm{~Hz} \rightarrow$ asystole with $500 \mu \mathrm{M} \mathrm{BPV} \rightarrow 2 \times 10^{-1}$ units $/ \mathrm{ml}$ insulin for $60 \mathrm{~min}$

2. Stable contractions at $1.2 \mathrm{~Hz} \rightarrow$ asystole with $500 \mu \mathrm{M} \mathrm{BPV} \rightarrow 33 \mathrm{mM}$ glucose and $2 \times 10^{-1}$ units $/ \mathrm{ml}$ insulin at $5 \mathrm{~min}$ after asystole for $60 \mathrm{~min}$

\section{RESULTS}

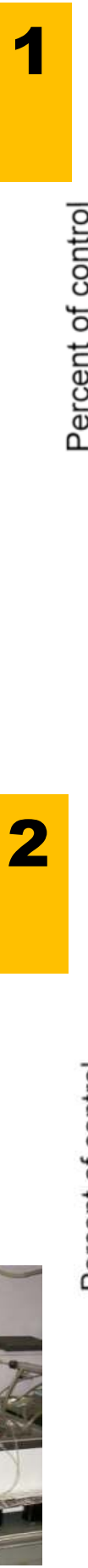

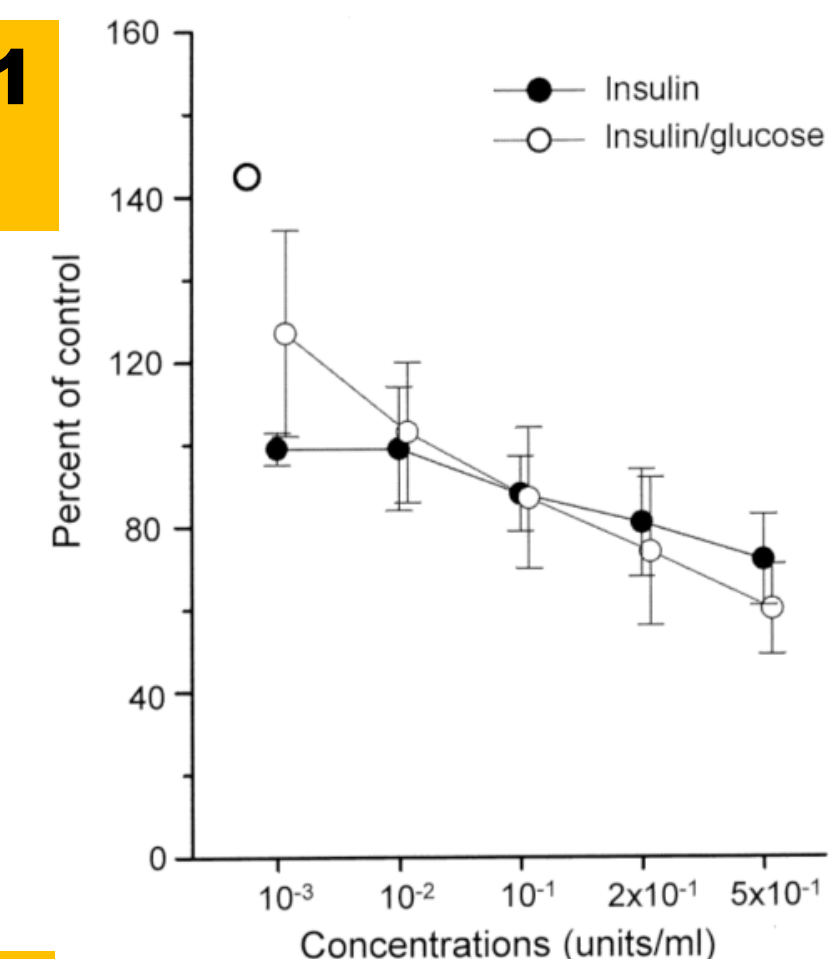

2

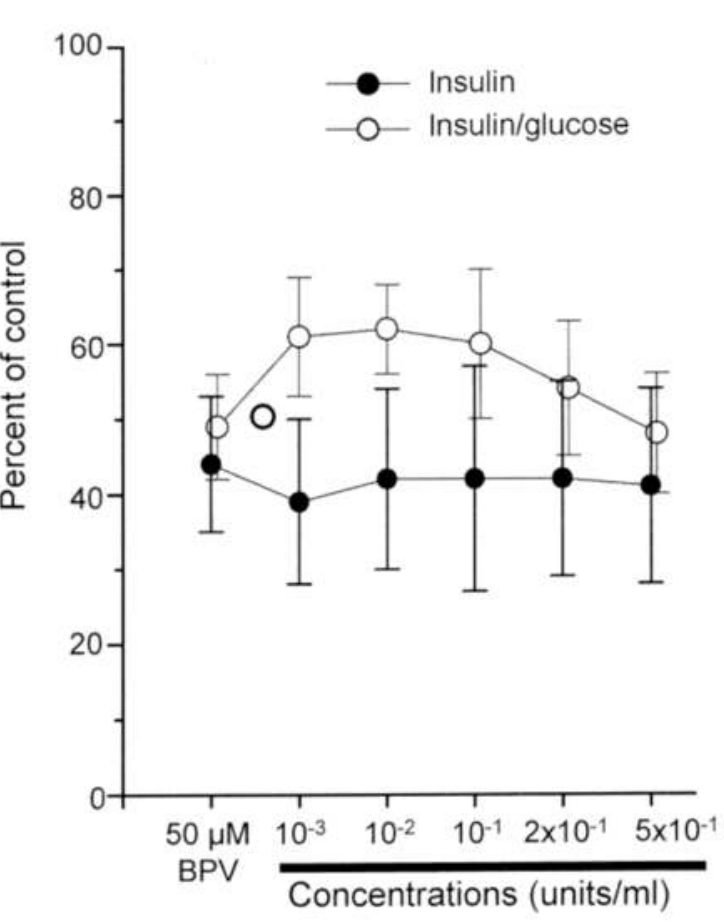

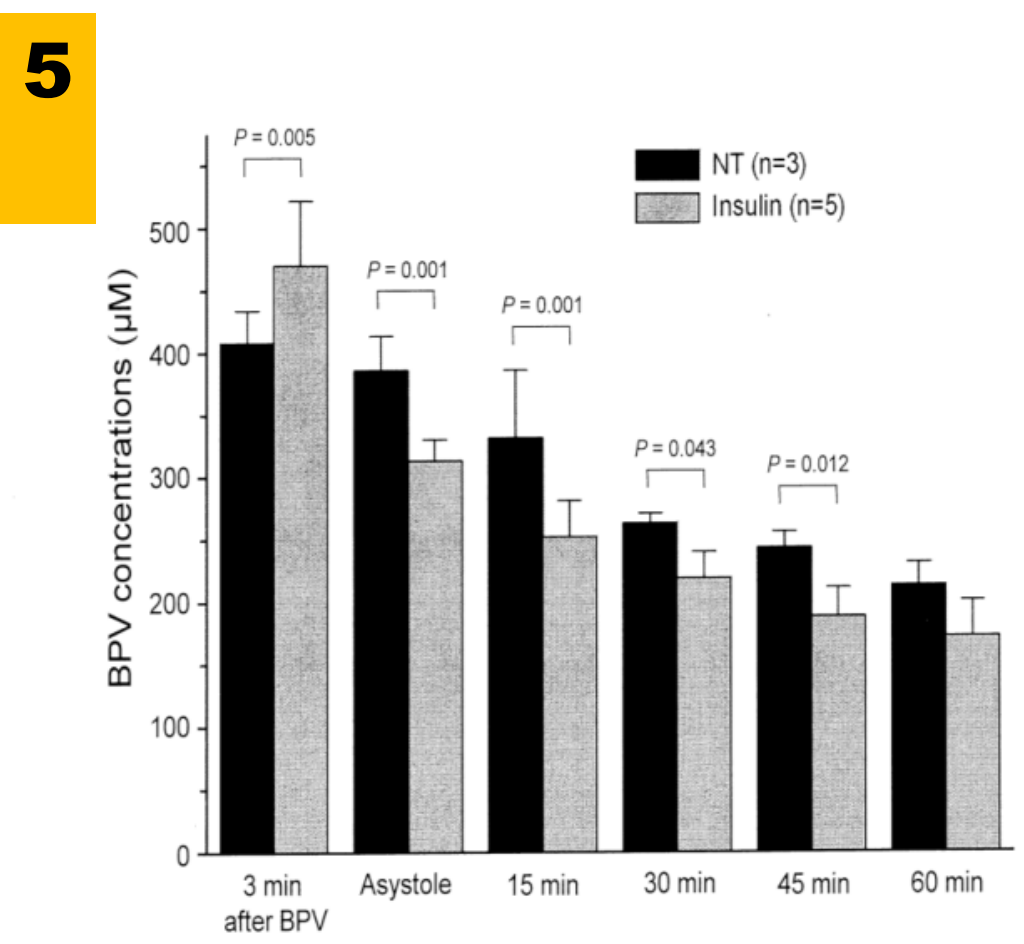

6

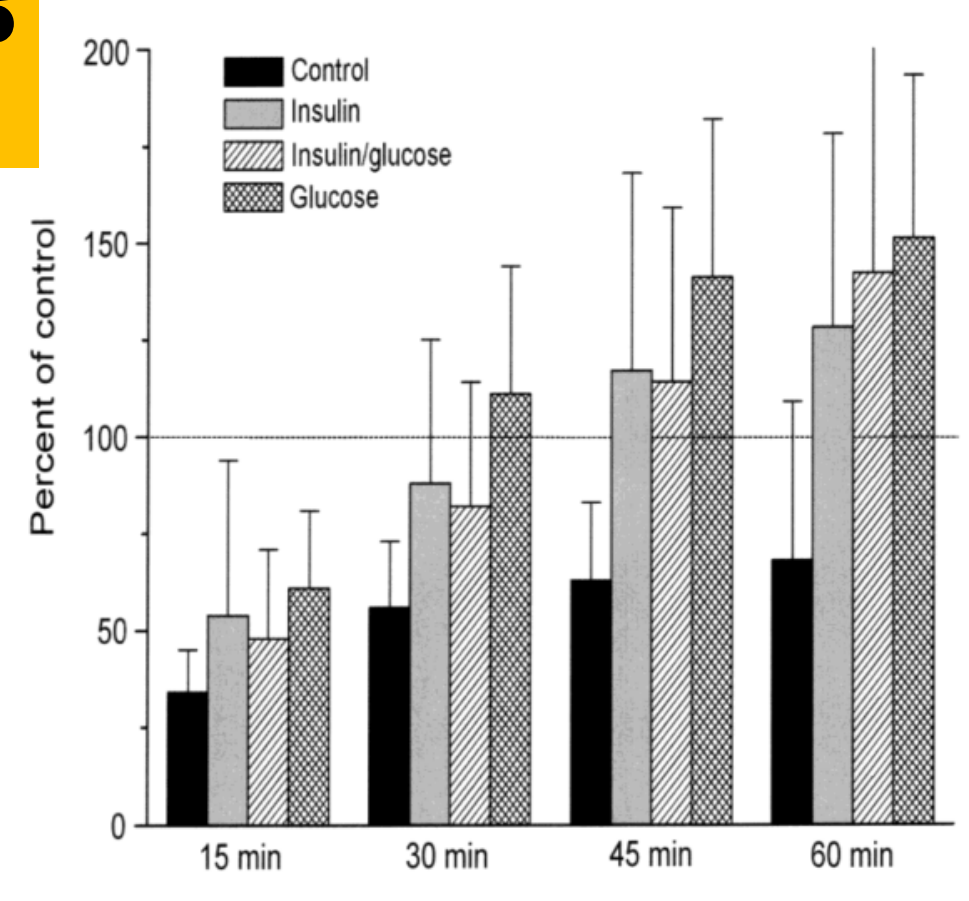

3

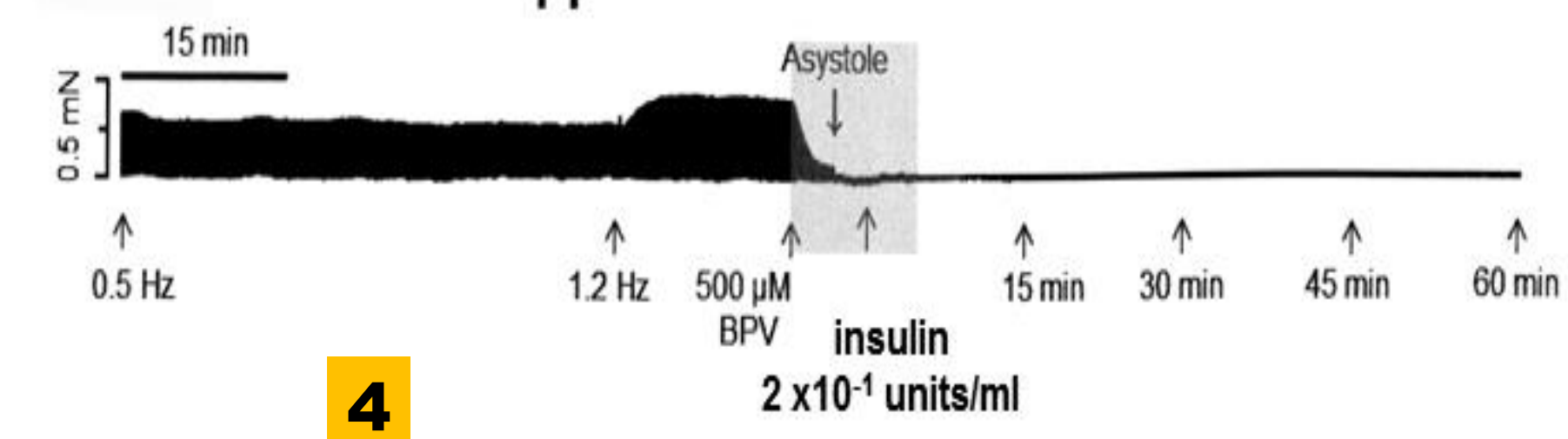

CONCLUSIONS

Neither insulin nor insulin/glucose treatment is likely to reverse BPVinduced cardiac conduction block and asystole in vitro, but, these treatments are likely to replenish the metabolic energy in the myocardium.

Circulatory support to eliminate BPV from the heart after BPVinduced cardiac arrest appears to be critical when treating with insulin and glucose. 